Цехмистро Л.Н.' , Фролов А.В. ${ }^{2}$, Мельникова О.П.'., Воробьев А.П. ${ }^{2}$

${ }^{1}$ Белорусский государственный университет физической культуры, Минск, Беларусь

${ }^{2}$ Республиканский научно-практический центр «Кардиология», Минск, Беларусь

Tsekhmistro L. ${ }^{1}$, Frolov A. ${ }^{2}$, Melnikova O. ${ }^{2}$, Vorobiev A. ${ }^{2}$

${ }^{1}$ Belarusian State University of Physical Culture, Minsk, Belarus

${ }^{2}$ Republican Scientific and Practical Centre "Cardiology", Minsk, Belarus

\title{
Сердечно-сосудистые риски
} у высококвалифицированных спортсменов на фоне субмаксимальных физических нагрузок

\author{
Cardiovascular Risks in Highly Qualified Athletes \\ on the Background of Sub-Maximum Physical Loads
}

Резюме

Цель. Исследовать динамику состояния сердечно-сосудистой системы высококвалифицированных спортсменов на фоне субмаксимальных физических нагрузок для идентификации лиц с высоким риском кардиоваскулярных осложнений, в том числе внезапной сердечной смерти.

Материалы и методы. Обследовано 134 спортсмена, из них 70 спортсменов циклических видов спорта, средний возраст 19,2 2,0 года, и 64 спортсмена ациклических видов, средний возраст 20,0 33,5 года. Контрольную группу составили 60 здоровых лиц, не занимающих-

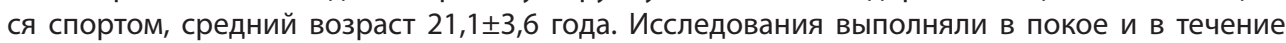
1-3 мин. после субмаксимальной физической нагрузки (200 кДж у мужчин и 140 кДж у женщин). Состояние сердечно-сосудистой системы оценивалось по динамике параметров ЭКГ высокого разрешения, отражающих электрическую нестабильность миокарда: альтернация Т-волны, длительность и дисперсия интервала QT, пространственный угол QRS-T, турбулентность сердечного ритма и торможение сердечного ритма. На основании измерений оценивали интегральный индекс электрической нестабильности миокарда.

Результаты. В состоянии покоя признаков электрической нестабильности миокарда не зафиксировано ни у спортсменов, ни у лиц контрольной группы. У высококвалифицированных спортсменов наблюдалась адекватная физиологическая реакция на физическую нагрузку, достоверно повысились систолическое АД и ЧСС ( $<<0,05)$. Статистически значимо повысилась альтернация Т-волны, уменьшился интервал QT, повысились дисперсия интервала QT, угол QRS-T, снизилось замедление сердечного ритма DC ( $<<0,05)$.

В переходном состоянии «нагрузка - покой» частота обнаружения ЭКГ-маркеров электрической нестабильности миокарда у спортсменов циклических видов спорта возросла в 2,9 раза, а у спортсменов ациклических видов - в 3,6 раза. Среди ЭКГ-маркеров электрической нестабильности наиболее чувствительными к нагрузкам оказались маркеры нестабильности в фазе реполяризации (альтернация Т-волны и дисперсия QT) и вагусная активность по замедлению сердечного ритма, 46,2 и 42,8\% соответственно.

Выводы. 1. В состоянии покоя у высококвалифицированных спортсменов не выявлены признаки электрической нестабильности миокарда. 2. После субмаксимальной физической 
нагрузки средний риск потенциальных кардиоваскулярных осложнений обнаружен только у 7,4\% спортсменов, случаев высокого риска не выявлено. 3. Существенных отличий между реакциями на физическую нагрузку у спортсменов циклических и ациклических видов спорта не обнаружено. 4. Чувствительность метода ЭКГ высокого разрешения при скрининге спортсменов в 3-4 раза выше, чем у традиционной ЭКГ в 12 стандартных отведениях.

Ключевые слова: кардиоваскулярные события, спортивная кардиология, электрическая нестабильность миокарда, электрокардиография высокого разрешения.

Abstract

Purpose. To study the dynamics of the state of the cardiovascular system of highly qualified athletes on the background of submaximal physical activity to identify persons with a high risk of cardiovascular events, including sudden cardiac death.

Materials and methods. 134 athletes were examined, of which 70 athletes were of cyclic sports,

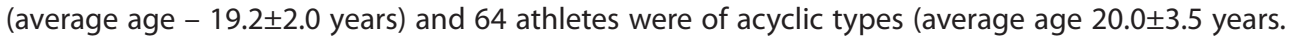
The control group consisted of 60 healthy people, who did not go in for sports (average age $21.1 \pm 3.6$ years. The studies were performed at rest and during 1-3 $\mathrm{min}$. after submaximal physical activity (200 kJ in men and $140 \mathrm{~kJ}$ in women). The state of the cardiovascular system was assessed by the dynamics of high-resolution ECG reflecting the electrical instability of the myocardium: T-wave alternation, duration and dispersion of the QT interval, spatial QRS-T angle, heart rate turbulence, and heart rate deceleration capacity. On the base of the measurements, the integral index of myocardial electrical instability was assessed too.

Results. At rest, no signs of electrical instability of the myocardium were recorded in either the athletes or the control group. Highly qualified athletes showed an adequate physiological response to physical activity; systolic blood pressure and heart rate significantly increased $(p<0.05)$. The T-wave alternation significantly increased, the QT interval decreased, the dispersion of the QT interval, the QRS-T angle increased, and the deceleration capacity DC of the heart rate decreased $(p<0.05)$. In the transitional state "load - rest", the frequency of detection of ECG markers of electrical instability of the myocardium in athletes of cyclic sports increased by 2.9 times and in athletes of acyclic types by 3.6 times. Among the ECG markers of electrical instability, the most sensitive to stress were the markers of instability in the repolarization phase (T-wave alternation and QT dispersion) and activity of vagal regulation according to heart rate deceleration capacity, 46.2 and $42.8 \%$, respectively.

Conclusions. 1. At rest, highly qualified athletes did not show the signs of electrical instability of the myocardium. 2. After submaximal physical activity, the average risk of potential cardiovascular events was found only in $7.4 \%$ of athletes; no cases of high risk were found. 3 . There were no significant differences between the responses to physical activity in athletes of cyclic and acyclic sports. 4. The sensitivity of the high-resolution ECG method for screening athletes is 3-4 times higher than that of the traditional ECG in 12 standard leads.

Keywords: cardiovascular events, sports cardiology, electrical instability of the myocardium, highresolution electrocardiography.

\section{- ВВЕДЕНИЕ}

Спортивная кардиология в первую очередь нацелена на обследование здоровых спортсменов. Тем не менее, в случае сочетания интенсивных физических и психоэмоциональных нагрузок и скрытой патологии со стороны сердечно-сосудистой системы возникает риск кардиоваскулярных осложнений, а в крайней форме - внезапной сердечной смерти (BCC). ВСС в профессиональном спорте крайне редка, 
однако периодически возникающие случаи получают большой общественный резонанс (недавний случай с игроком футбольной сборной Дании на Евро-2020). Оценки частоты ВСС среди атлетов колеблются от 1:1 000000 до 1:500 000, такой разброс обусловлен отсутствием до недавних пор стандартизированной методологии учета и разнородностью групп обследования. В когорту спортсменов с наиболее высоким риском ВСС в США входят баскетболисты негроидной расы, а в Европе - профессиональные футболисты [1]. В 90-95\% случаев причинами ВСС в спорте являются желудочковые тахиаритмии (желудочковая тахикардия / фибрилляция желудочков), поэтому соответствующие технологии скрининга должны быть в первую очередь нацелены на прогнозирование данной патологии. С целью минимизации риска ВСС в странах ЕС и США проводятся регулярные скрининговые обследования спортсменов, разрабатываются соответствующие международные стандарты $[2,3]$. Например, в Италии внедрение обязательного ЭКГскрининга при допуске к спортивным состязаниям как на профессиональном, так и на любительском уровне позволило за 25 лет снизить частоту ВСС среди спортсменов с 4 до 0,4/100 000 случаев в год, хотя ранее данный показатель был в 3 раза выше, чем у сверстников, не занимающихся спортом [4, 5].

Вместе с тем диагностика ранних стадий атеросклероза, некоронарной кардиомиопатии, аритмогенной дисплазии правого желудочка и иной структурной патологии по данным классической ЭКГ практически невозможна, а привлечение визуальных методов диагностики (КT, МРТ) на этапе массового скрининга спортсменов нецелесообразно по экономическим соображениям. Вследствие этого разработка более чувствительных в отношении прогнозирования жизненно опасных тахиаритмий и одновременно неинвазивных и экономичных технологий скрининга важна не только для профессионального спорта, но и для оздоровления населения в целом.

\section{- ЦЕЛЬ ИССЛЕДОВАНИЯ}

Исследование динамики состояния сердечно-сосудистой системы высококвалифицированных спортсменов на фоне субмаксимальных физических нагрузок для идентификации лиц с высоким риском кардиоваскулярных осложнений, в том числе внезапной сердечной смерти.

\section{МАТЕРИАЛЫ И МЕТОДЫ}

Обследовано 134 спортсмена высокой спортивной квалификации (мастера спорта, кандидаты в мастера спорта). В 1-ю группу включили 70 спортсменов циклических видов спорта (гребля, велоспорт), средний возраст 19,2 2,0 года, во 2-ю группу - 64 спортсмена ациклических

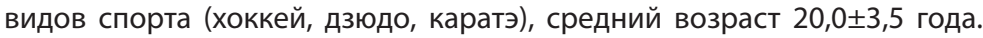
Контрольную группу составили 60 молодых здоровых лиц, не занимающихся спортом, средний возраст 21,1土3,6 года. Исследование сердечнососудистой системы (ССС) проводили как в состоянии покоя, так и после субмаксимальной специфической спортивной нагрузки. Спортсмены циклических видов выполняли велоэргометрический тест с начальной мощностью 125 Вт (мужчины) или 90 Вт (женщины), длительностью каждой ступени 2 мин., приращением мощности на 30 Вт (муж.) или 20 Вт 
(жен.), всего 7-8 циклов. При этом объем работы для мужчин составил в среднем 200 кДж, а для женщин - 140 кДж соответственно. Спортсменам ациклических видов назначали 60-минутную тренировку скоростно-силовой направленности. Измерения состояния ССС выполняли не позднее 1-3 мин. после нагрузки. Первичными конечными точками считались желудочковые нарушения сердечного ритма и синкопальные состояния.

В основе патологических механизмов фатальных тахиаритмий лежат как структурные, так и электрические дисфункции миокарда. Полезной прогностической информацией в отношении тахиаритмий обладает электрическая нестабильность миокарда, которая проявляется в beatto-beat изменениях потенциала действия кардиомиоцитов и особенно его заднего фронта. На электрофизиологическом уровне эти изменения трансформируются в микроальтернации амплитудных и временных параметров ЭКГ. Высокая степень альтернации ЭКГ-маркеров признана предиктором жизненно опасных аритмий и ВСС [6, 7]. Оценка маркеров нестабильности миокарда доступна при применении ЭКГ высокого разрешения.

Нами использован 12-канальный цифровой электрокардиограф «Интекард» со специализированным программным обеспечением (разработка РНПЦ «Кардиология» и УП «Кардиан»). Кроме традиционной синдромальной диагностики с помощью программы «Интекард 7.3» оценивался ряд ЭКГ-маркеров электрической нестабильности миокарда (ЭНМ) в соответствии с требованиями международных стандартов измерения и интерпретации [8-12]. Среди них альтернация Т-волны, длительность и дисперсия интервала QT, пространственный угол QRS-T, турбулентность сердечного ритма и торможение сердечного ритма. Альтернация T-волны (TWA) и дисперсия интервала QT (dQT) вместе указывают на ЭНМ в фазе реполяризации при превышении пороговых значений TWA >45 мкB и dQT >70 мс соответственно. Угол QRS-T, превышающий порог $105^{\circ}$, отражает нарушение синергичности между процессами де- и реполяризации. С помощью турбулентности сердечного ритма (HRT) выявляется нарушение барорецепторного контроля сердечного ритма, пороговые значения TO $>0 \%$ и/или TS $<15$ мс/RR, где TO - начало турбулентности, TS - наклон турбулентности, RR - кардиоинтервал в Mc. Замедление сердечного ритма (DC) отражает депрессию вагусного отдела вегетативной регуляции при $D C<4,5$ мс. На основе измерений вышеуказанных ЭКГ-маркеров рассчитывался интегральный индекс электрической нестабильности миокарда [13]. Кроме того, у спортсменов и лиц контрольной группы измеряли артериальное давление (АД), вариабельность сердечного ритма и при необходимости проводили ЭхоКГ.

Результаты исследования представлены в виде $\mathrm{M} \pm \mathrm{SD}$ или $\mathrm{Me}(\mathrm{Q} 25$, Q75) в зависимости от типа распределения. Проверку нормальности распределения выполняли с помощью критерия Шапиро - Уилка. При анализе различий между группами использовали t-критерий Стьюдента, критерий $X^{2}$ Пирсона или U-критерий Манна - Уитни в зависимости от типа распределения. Критическое значение уровня статистической значимости при проверке нулевых гипотез принималось равным 0,05. Обработка данных выполнена с помощью прикладных пакетов Statistica, v.10 (Stat Soft) и SPSS Statistics, v.23 (IBM). 


\section{— РЕЗУЛЬТАТЫ И ОБСУЖДЕНИЕ}

Выполнено исследование функционального статуса ССС у спортсменов циклических и ациклических видов спорта, а также у лиц контрольной группы в состоянии покоя. Полученные данные представлены в табл. 1.

Из табл. 1 следует, что у лиц контрольной группы параметры центральной гемодинамики (АД сист., АД диаст., ЧСС) находятся в пределах физиологической нормы и статистически значимо не отличаются от аналогичных показателей у спортсменов. Признаков ЭНМ в покое не зафиксировано ни у спортсменов, ни у лиц контрольной группы.

Статистически значимые различия зафиксированы при сравнении параметров, отражающих статус вегетативной нервной системы. У спортсменов ациклических видов статистически значимо повысилась высокая частота спектра HF, что указывает на доминирование вагусного отдела вегетативной регуляции $(p=0,012)$. Кроме того, в контрольной группе было значимо выше значение стресс-индекса Si, чем у

\section{Таблица 1}

Параметры центральной гемодинамики и маркеры электрической нестабильности миокарда у спортсменов циклических и ациклических видов спорта и лиц контрольной группы в покое

\begin{tabular}{|c|c|c|c|c|}
\hline Показатели & $\begin{array}{l}\text { Контрольная } \\
\text { группа } \\
(\mathrm{n}=60)\end{array}$ & $\begin{array}{l}\text { Циклические } \\
\text { виды } \\
\text { (n=70) }\end{array}$ & $\begin{array}{l}\text { Ациклические } \\
\text { виды } \\
(n=64)\end{array}$ & Уровень P \\
\hline $\begin{array}{l}\text { АД сист., } \\
\text { мм рт. ст. }\end{array}$ & $114,4 \pm 10,9$ & $115,9 \pm 7,3$ & $113,9 \pm 6,7$ & $\begin{array}{l}1,2-0,430 \\
1,3-0,841\end{array}$ \\
\hline $\begin{array}{l}\text { АД диаст., } \\
\text { мм рт. ст. }\end{array}$ & $74,0 \pm 7,4$ & $72,1 \pm 7,2$ & $71,8 \pm 7,5$ & $\begin{array}{l}1,2-0,238 \\
1,3-0,234\end{array}$ \\
\hline ЧСС, мин ${ }^{-1}$ & $69,4 \pm 10,7$ & $71,5 \pm 12,7$ & $64,5 \pm 10,6$ & $\begin{array}{l}1,2-0,419 \\
1,3-0,065\end{array}$ \\
\hline Альтернация Т, мкВ & $22(17 ; 25)$ & $21(16,5 ; 24,5)$ & $21(17 ; 25)$ & $\begin{array}{l}1,2-0,467 \\
1,3-0,795\end{array}$ \\
\hline Интервал QT, мс & $364,1 \pm 24,4$ & $364,5 \pm 31,7$ & $375,0 \pm 27,5$ & $\begin{array}{l}1,2-0,953 \\
1,3-0,089 \\
\end{array}$ \\
\hline Дисперсия QT, мс & $47,3 \pm 13,8$ & $46,7 \pm 17,3$ & $48,5 \pm 13,7$ & $\begin{array}{r}1,2-0,858 \\
1,3-0,734\end{array}$ \\
\hline Угол QRS-T, ${ }^{\circ}$ & $68,4 \pm 23,1$ & $66,4 \pm 23,8$ & $58,7 \pm 24,6$ & $\begin{array}{l}1,2-0,694 \\
1,3-0,101 \\
\end{array}$ \\
\hline $\begin{array}{l}\text { Замедление ритма } \\
\text { DC, мC }\end{array}$ & $16(9,8 ; 22,4)$ & $20,8(13,3 ; 33,0)^{*}$ & $19,6(14,0 ; 28,8)$ & $\begin{array}{l}1,2-0,045 \\
1,3-0,066 \\
\end{array}$ \\
\hline SDNN, MC & $88,7 \pm 37,8$ & $100,1 \pm 32,1$ & $95,9 \pm 40,0$ & $\begin{array}{l}1,2-0,099 \\
1,3-0,419 \\
\end{array}$ \\
\hline Si, усл. ед. & $246 \pm 175$ & $169 \pm 93^{*}$ & $220 \pm 133$ & $\begin{array}{l}\mathbf{1}, \mathbf{2}-\mathbf{0 , 0 0 3} \\
1,3-0,468\end{array}$ \\
\hline$H F, \%$ & $60,5 \pm 4,4$ & $61,1 \pm 5,8$ & $63,2 \pm 4,7^{*}$ & $\begin{array}{l}1,2-0,579 \\
\mathbf{1 , 3} \mathbf{3} \mathbf{0 , 0 1 2} \\
\end{array}$ \\
\hline$L F, \%$ & $31,0 \pm 3,1$ & $30,9 \pm 4,1$ & $29,4 \pm 4,3$ & $\begin{array}{l}1,2-0,948 \\
1,3-0,078\end{array}$ \\
\hline VLF, \% & $8,5 \pm 2,7$ & $8,0 \pm 2,8$ & $7,4 \pm 1,7^{*}$ & $\begin{array}{l}1,2-0,322 \\
\mathbf{1 , 3 - 0 , 0 2 8}\end{array}$ \\
\hline
\end{tabular}

Примечания: $\mathrm{p}_{1,2}$ - уровень значимости различий между контрольной группой и группой спортсменов циклических видов спорта; $\mathrm{p}_{1,3}$ - уровень значимости различий между контрольной группой и группой спортсменов ациклических видов спорта, SDNN - среднее квадратичное отклонение RR-интервалов, Si - стресс-индекс, HF - высокая частота спектра, LF - низкая частота спектра, VLF - очень низкая частота спектра, * $p<0,05$. 
Научные публикации. Оригинальные исследования

Table 1

Central hemodynamic parameters and markers of myocardial electrical instability in athletes of cyclic and acyclic sports and individuals in the control group at rest

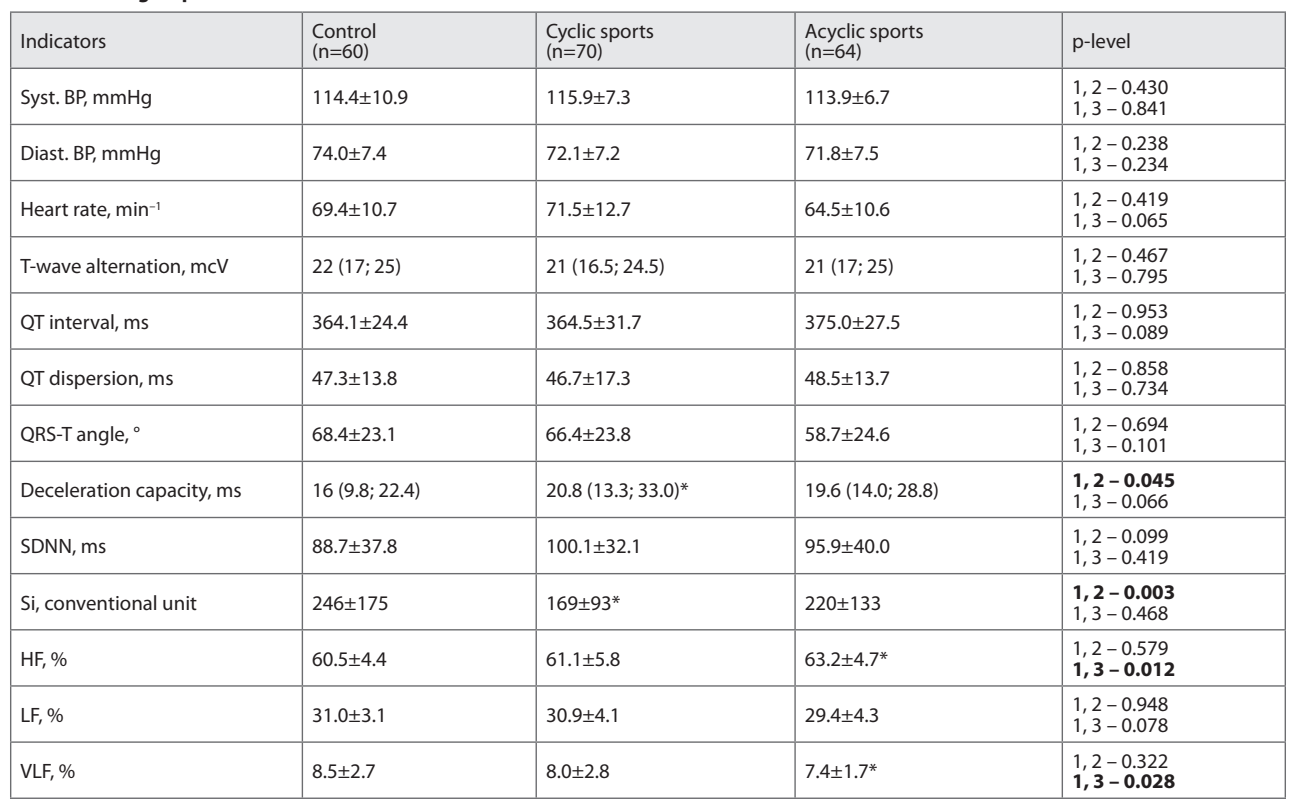

Notes: p1, 2 - the level of significance of differences between the control group and the group of athletes in cyclic sports; p1, 3 - the level of significance of differences between the control group and the group of athletes of acyclic sports, SDNN - standard deviation of RR-intervals, Si - stress index, HF - high frequency of the spectrum, LF - low frequency of the spectrum, VLF - very low frequency of the spectrum, ${ }^{*} p<0.05$.

спортсменов циклических видов ( $p=0,003)$. Этот маркер ассоциируется с централизацией вегетативной нервной регуляции.

Проведено исследование динамики состояния ССС у спортсменов после выполнения ими субмаксимальной физической нагрузки. У лиц контрольной группы нагрузочные тесты не проводились по причине низкой толерантности к нагрузкам субмаксимальной мощности, а сравнение с нагрузками более низкой мощности представляется некорректным. Полученные результаты представлены в табл. 2.

У высококвалифицированных спортсменов наблюдается адекватная физиологическая реакция на субмаксимальную нагрузку. Статистически достоверно повысились систолическое АД и чСС $(p<0,05)$. У спортсменов ациклических видов незначимо повысилось диастолическое АД. Среди ЭКГ-маркеров ЭНМ статистически значимо повысилась альтернация Т-волны (в группе циклических видов спорта), уменьшился интервал QT, повысились дисперсия интервала QT и угол QRS-T $(p<0,05)$. Кроме того, снизилось замедление сердечного ритма DC, что указывало на доминирование в постнагрузочном периоде симпатоадреналового отдела вегетативной регуляции над вагусным. У спортсменов циклических видов спорта было превышено групповое пороговое значение дисперсии интервала QT 70 мс, по остальным ЭКГ-маркерам ЭНМ превышений пороговых значений не было зафиксировано.

В постнагрузочный период значения корригированного по Базетту интервала QTкор. не превысили порог физиологической нормы 440 Mc, 
Сердечно-сосудистые риски у высококвалифицированных спортсменов на фоне субмаксимальных физических нагрузок

\section{Таблица 2}

Динамика центральной гемодинамики и маркеров электрической нестабильности миокарда в переходном состоянии «нагрузка - покой» у высококвалифицированных спортсменов

\begin{tabular}{|c|c|c|c|c|}
\hline \multirow[b]{2}{*}{ Параметры } & \multicolumn{2}{|c|}{ Циклические виды спорта } & \multicolumn{2}{|c|}{ Ациклические виды спорта } \\
\hline & $\begin{array}{l}\text { Состояние } \\
\text { покоя }\end{array}$ & $\begin{array}{l}\text { После } \\
\text { нагрузки }\end{array}$ & $\begin{array}{l}\text { Состояние } \\
\text { покоя }\end{array}$ & $\begin{array}{l}\text { После } \\
\text { нагрузки }\end{array}$ \\
\hline АД сист., мм рт. ст. & $115,9 \pm 7,3$ & $186,2 \pm 20,7^{*}$ & $113,9 \pm 6,7$ & $172 \pm 44,1^{*}$ \\
\hline АД диаст., мм рт. ст. & $72,1 \pm 7,2$ & $94,5 \pm 17,0^{*}$ & $71,8 \pm 7,5$ & $76,4 \pm 26,4$ \\
\hline ЧСС, мин ${ }^{-1}$ & $71,5 \pm 12,7$ & $107,1 \pm 30,8^{*}$ & $64,5 \pm 10,6$ & $118,7 \pm 18,5^{*}$ \\
\hline Альтернация T, мкВ & $21(16,5 ; 24,5)$ & $24(17 ; 37)^{*}$ & $21(17 ; 25)$ & $22(18 ; 28)$ \\
\hline Интервал QT, мс & $364,5 \pm 31,7$ & $331,4 \pm 44,4^{*}$ & $375 \pm 27,5$ & $308,3 \pm 17^{*}$ \\
\hline Дисперсия QT, мс & $46,7 \pm 17,3$ & $82,3 \pm 37,3^{*}$ & $48,5 \pm 13,7$ & $71,9 \pm 22,8^{*}$ \\
\hline Угол QRS-T, ${ }^{\circ}$ & $66,4 \pm 23,8$ & $83,6 \pm 26,8^{*}$ & $58,7 \pm 24,6$ & $79,1 \pm 28^{*}$ \\
\hline Замедление DC, мc & $20,8(13,3 ; 33)$ & $4,3(2,5 ; 10,3)$ & $19,6(14 ; 28,8)$ & $4,3(2,5 ; 5,3)^{*}$ \\
\hline
\end{tabular}

Примечание: * $\mathrm{p}<0,05$.

Table 2

Dynamics of central hemodynamics and markers of electrical instability of the myocardium in the transitional state "load - rest" in highly qualified athletes

\begin{tabular}{|l|l|l|l|l|}
\hline \multirow{2}{*}{ Indicators } & \multicolumn{2}{l|}{ Cyclic sports } & \multicolumn{2}{l|}{ Acyclic sports } \\
\cline { 2 - 5 } & At rest & After load & At rest & After load \\
\hline Syst. BP, $\mathrm{mmHg}$ & $115.9 \pm 7.3$ & $186.2 \pm 20.7^{*}$ & $113.9 \pm 6.7$ & $172 \pm 44.1^{*}$ \\
\hline Diast. BP, $\mathrm{mmHg}$ & $72.1 \pm 7.2$ & $94.5 \pm 17.0^{*}$ & $71.8 \pm 7.5$ & $76.4 \pm 26.4$ \\
\hline Heart rate, min ${ }^{-1}$ & $71.5 \pm 12.7$ & $107.1 \pm 30.8^{*}$ & $64.5 \pm 10.6$ & $118.7 \pm 18.5^{*}$ \\
\hline T-wave alternation, mcV & $21(16.5 ; 24.5)$ & $24(17 ; 37)^{*}$ & $21(17 ; 25)$ & $22(18 ; 28)$ \\
\hline QT interval, ms & $364.5 \pm 31.7$ & $331.4 \pm 44.4^{*}$ & $375 \pm 27.5$ & $308.3 \pm 17^{*}$ \\
\hline QT dispersion, ms & $46.7 \pm 17.3$ & $82.3 \pm 37.3^{*}$ & $48.5 \pm 13.7$ & $71.9 \pm 22.8^{*}$ \\
\hline QRS-T angle, ${ }^{\circ}$ & $66.4 \pm 23.8$ & $83.6 \pm 26.8^{*}$ & $58.7 \pm 24.6$ & $79.1 \pm 28^{*}$ \\
\hline Deceleration capacity, ms & $20.8(13.3 ; 33)$ & $4.3(2.5 ; 10.3)$ & $19.6(14 ; 28.8)$ & $4.3(2.5 ; 5.3)^{*}$ \\
\hline
\end{tabular}

Note: ${ }^{*} \mathrm{p}<0.05$.

то есть синдром удлиненного QT у спортсменов не обнаружен. Значения турбулентности сердечного ритма не определены, так как у спортсменов не были зафиксированы желудочковые экстрасистолы.

Для всех обследованных спортсменов выполнен анализ индивидуальных реакций ССС на специфические субмаксимальные нагрузки. Для каждого испытуемого спортсмена были отмечены превышения пороговых значений маркеров ЭНМ как в состоянии покоя, так и после выполнения нагрузки.

Так, среди спортсменов циклических видов спорта ( $\mathrm{n}=70)$ в покое у 49 (70,0\%) испытуемых не были зафиксированы превышения пороговых значений ЭКГ-маркеров ЭНМ, у 19 (27,2\%) испытуемых выявлены превышения по 1 маркеру ЭНМ и у 2 (2,8\%) - превышения пороговых значений по 2 маркерам ЭНМ. Синхронное превышение по 3 и 4 маркерам не зафиксировано. В этой же группе после выполнения нагрузки частота выявления маркеров ЭНМ возросла. У 28 (40,0\%) испытуемых не зафиксированы превышения маркеров ЭНМ, у 17 (24,3\%) испытуемых выявлены превышения пороговых значений по 1 маркеру эНМ, у 19 (27,1\%) - превышения по 2 маркерам ЭНМ, а у 6 (8,6\%) - превышения по 3 маркерам ЭНМ. Таким образом, в переходном состоянии «нагрузка - покой» частота обнаружения ЭКГ-маркеров ЭНМ у спортсменов циклических видов спорта возросла в 2,9 раза. 
В группе спортсменов ациклических видов спорта $(\mathrm{n}=64)$ в состоянии покоя у 44 (68,7\%) обследованных не были зафиксированы превышения порогов ЭКГ-маркеров ЭНМ, у 18 (28,1\%) - выявлены превышения по 1 маркеру ЭНМ, у 2 (3,2\%) - по 2 маркерам ЭНМ. В переходном состоянии «нагрузка - покой» частота обнаружения ЭКГ-маркеров ЭНМ также возросла. Всего у 10 (15,6\%) испытуемых не зафиксированы превышения пороговых значений маркеров ЭНМ, у 33 (51,6\%) испытуемых обнаружены превышения по 1 маркеру, у 17 (26,6\%) - по 2 маркерам, а у 4 (6,2 \%) - по 3 маркерам ЭНМ. То есть в данной группе спортсменов частота обнаружения ЭКГ-маркеров ЭНМ после нагрузки возросла в 3,6 раза.

Для выявления ЭКГ-маркеров ЭНМ, наиболее чувствительных к воздействию субмаксимальных физических нагрузок, определяли их частоту в восстановительном периоде «нагрузка - покой». На рис. 1 демонстрируется частота обнаружения ЭКГ-маркеров QRS-T, dQT, TWA и DC в восстановительном периоде. Нарушение синергичности процессов деполяризации/реполяризации миокарда, определяемое по значению угла QRS-T $>105^{\circ}$, составило 11,0\%. Аддитивная частота электрической нестабильности в фазе реполяризации, выявленная по дисперсии dQT $>70$ мс и альтернации Т-волны TWA >45 мкВ, в сумме составила 46,8\%. Наконец, доля дисфункций со стороны вегетативной нервной регуляции по данным замедления сердечного ритма $D C<4,5$ мс оказалась равной 42,8\%. Из этого следует, что субмаксимальные физические нагрузки в основном влияют на фазу реполяризации миокарда и приводят к доминированию симпатоадреналового отдела вегетативной нервной регуляции.

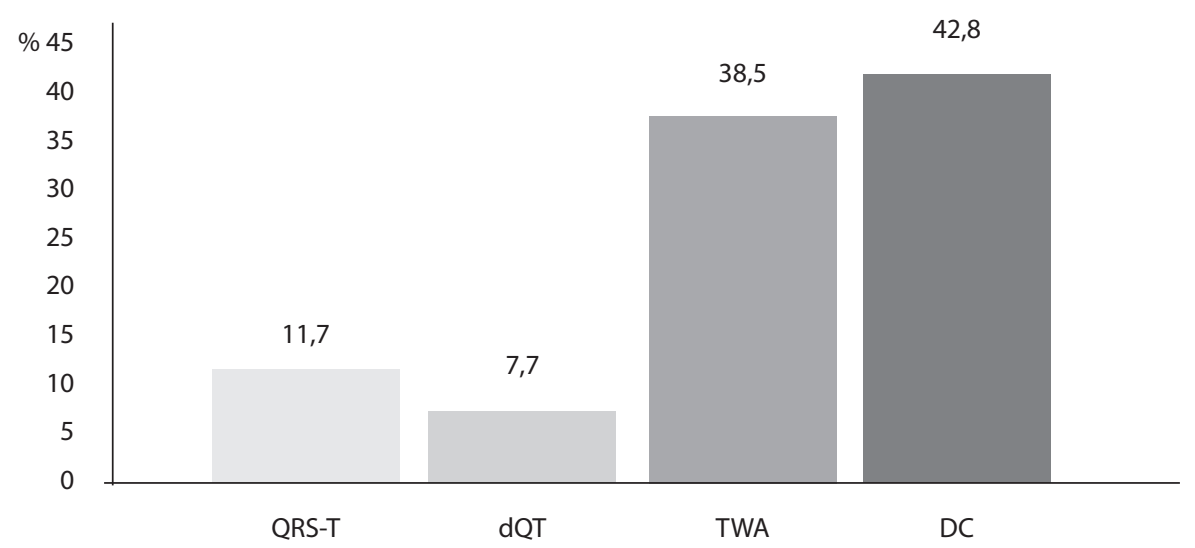

Рис. 1. Частота обнаружения ЭКГ-маркеров электрической нестабильности миокарда у спортсменов после субмаксимальной физической нагрузки

Примечания: QRS-T - пространственный угол между векторами QRS и T, dQT - дисперсия интервала QT, TWA - альтернация Т-волны, DC - замедление сердечного ритма.

Fig. 1. Detection rate of ECG-markers of electrical myocardial instability in athletes after submaximal physical load

Notes: QRS-T - 3D angle between QRS and T vectors, dQT - dispersion of the QT interval, TWA - T-wave alternation, DC - deceleration capacity. 

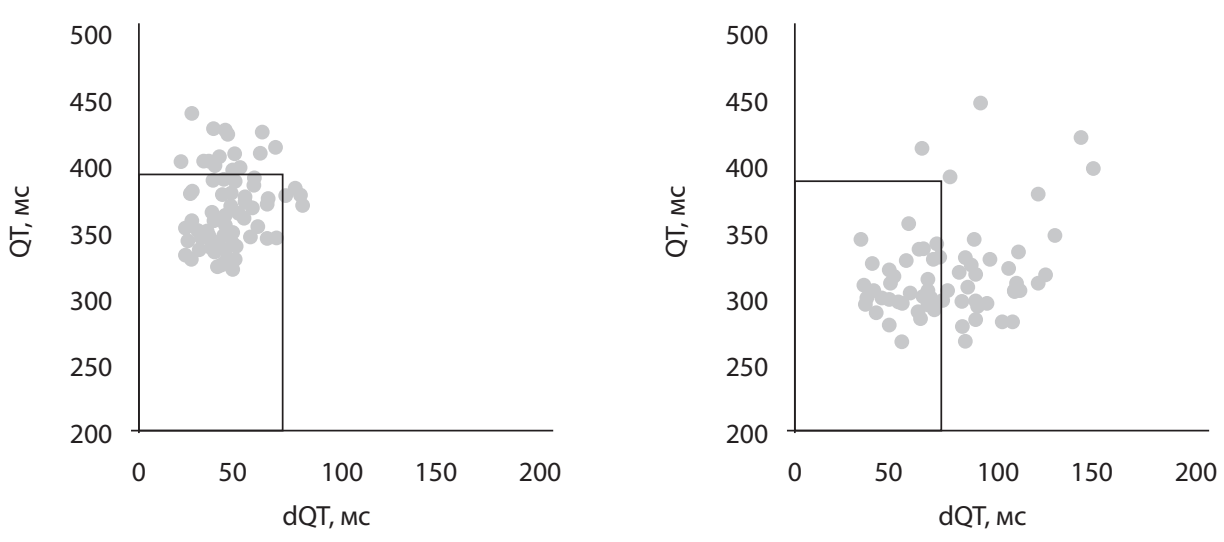

Рис. 2. Полигон рассеяния интервала и дисперсии QT в покое (слева) и после физической нагрузки (справа)

Fig. 2. The scattering polygon of the interval and dispersion QT at rest (left) and after physical load (right)

Установлен феномен повышения степени хаотичности в фазе реполяризации миокарда, который визуально представлен на рис. 2. Это полигон рассеяния самого интервала и дисперсии интервала QT в покое (слева) и после физической нагрузки (справа). Линейными отрезками отмечены граничные значения для интервала QT 395 мс и дисперсии dQT $70 \mathrm{MC}$.

Наглядно видно увеличение разброса распределения точек в постнагрузочном периоде в сравнении с состоянием покоя. Большой разброс косвенно свидетельствует об индивидуальном характере реакций ССС каждого спортсмена на нагрузку.

Риск-стратификация кардиоваскулярных осложнений у спортсменов по данным интегрального индекса ЭНМ дала следующие результаты. Полное отсутствие кардиоваскулярного риска установлено у $65,7 \%$ спортсменов, низкий риск - у 26,9\%, средний риск - у 7,4\%, высокая степень риска не обнаружена. При этом средний риск кардиоваскулярных событий у спортсменов не следует однозначно относить к патологическим. Процессы электрической неустойчивости миокарда могут проявляться при перетренированности, усталости, травмах или общих соматических недомоганиях. Тем не менее, на тех спортсменов, у которых выявлено по 3 и более ЭКГ-маркеров ЭНМ, должно быть сконцентрировано повышенное внимание со стороны тренеров и спортивных медиков [14]. Тем самым на этапе ЭКГ-скрининга можно проводить первичную профилактику ВСС среди спортсменов.

Таким образом, применение ЭКГ высокого разрешения при скрининге спортсменов позволяет сократить число ложноположительных нагрузочных тестов и выявить лиц с высоким риском ВСС с сохранением опций высокой пропускной способности и экономичности.

\section{ВЫВОДЫ}

1. В состоянии покоя у высококвалифицированных спортсменов не выявлены признаки электрической нестабильности миокарда. 
2. После субмаксимальной физической нагрузки средний риск потенциальных кардиоваскулярных осложнений обнаружен у 7,4\% спортсменов, случаев высокого риска не выявлено.

3. Существенных отличий между реакциями на физическую нагрузку у спортсменов циклических и ациклических видов спорта не обнаружено.

4. Чувствительность метода ЭКГ высокого разрешения при скрининге спортсменов в 3-4 раза выше, чем у традиционной ЭКГ в 12 стандартных отведениях.

Статья подготовлена в рамках задания № 13 ГНТП «Физическая культура и спорт» на 2021-2025 гг.

Вклад авторов: Цехмистро Л.Н. - набор материала исследования, формулировка выводов; Фролов А.В. - концепция проекта, интерпретация результатов; Мельникова О.П. - обработка результатов; Воробьев А.П. - разработка дизайна исследования, программное обеспечение.

Authors' contribution: Tsekhmistro L. - collection of research material, formulation of conclusions; Frolov A. - project concept, interpretation of results; Melnikova O. - processing of results; Vorobiev A. - research design development, software.

Конфликт интересов. Авторы заявляют об отсутствии конфликта интересов.

Conflict of interest. The authors declare no conflict of interest.

\section{ЛИТЕРATУPA/REFERENCES}

Harmon K., Maleszevski J., Owens D. (2015) Comparison of cardiovascular screening in college athletes by history and physical Incidence, cause, and comparative frequency of sudden cardiac death in national collegiate athletic association: a decade in review. Circulation, vol. 132, pp. 10-19.

2. Corrado D., Pellicia A., Bjornstad H. (2005) Cardiovascular pre-participation screening of young athletes for prevention of sudden cardiac death. Proposal for a common European protocol. Consensus statement of the study group of sport cardiology of the working group of myocardial and pericardial disease of the European Society of cardiology. European Heart Journal, vol. 28, pp. 518-524.

3. Pellicia A., Sharma S., Gati S. (2021) Guidelines on sport cardiology and exercise in patients with cardiovascular disease of the European Society of Cardiology (ESC). European Heart Journal, vol. 42 (1), pp. 5-6. doi: 10.1093/eurheartj/ehaa734.

4. Corrado D., Basso C., Pavei A. (2006) Trends in sudden cardiac death in young competitive athletes after implementation of participation screening program. JAMA, vol. 296, pp. 296-301.

5. Basso C., Rizzo S., Carturan E. (2020) Cardiac arrest at rest and during sport activity: causes and prevention. European Heart Journal, vol. 22 (Suppl. E), pp. 20-24. doi: 10..1093/ eurheartj/suaa052

6. Lampert R. (2015) ECG signature of physiological stress. Journal of Electrocardiology, vol. 48 (6), pp. 1000-1005. doi: 10.1016/j.electrocard.2015.08.005.

7. Frolov A., Vaikhanskaya T., Melnikova O. (2017) Risk-stratification personalized model for prediction of life-threatening ventricular tachyarrhythmias in patients with chronic Frolov A., Vaikhanskaya T., Melnikova O. (2017) Risk-stratification personalized model for prediction of life-threatening ventricular tachyarrhythmias in patients with chronic
heart failure. Kardiologia Polska, vol. 75 (7), pp. 682-688. doi: 10.5603/KP. a2017.0060.

8. Verrier R., Kingenheben T., Malik M. (2011) Microvolt T-wave alternans. Physiological basic, methods of measurement, and clinical utility consensus. J. of the Am. Coll. Cardiol, vol. 58 (13), pp. 1309-1324. doi: 10.1016/jacc.2011.06.029.

9. Malik M., Batchvarov V. (2000) Measurement, interpretation, and clinical potential of QT interval dispersion. J. of the Am. Coll. Cardiol, vol. 36 (6), pp. 1749-1766. doi: 10.1016/50735-1097(00)00962-1

10. Bauer A., Malik M., Schmidt G., Barthel P. (2008) Heart rate turbulence: standards of measurement, physiological interpretation, and clinical use. J. of the Am. Coll. Cardiol, vol. 52 (17), pp. 1353-1365. doi: 10.1016/jacc.2008.07.041.

11. Bauer A., Kantelhardt J., Barthel P., Schoming A. (2006) Deceleration capacity of heart rate as a predictor of mortality after myocardial infarction: cohort study. Lancet, vol. 367, pp. 1674-1681.

12. Kardys I., Kors J., van der Meer I. (2003) Spatial QRS-T angle predicts cardiac death in general population. Eur. Heart J, vol. 24 (14), pp. 1357-1364. doi: 10.1016/SO195668X(03)00203-3.

13. Frolov A., Vajhanskaya T., Melnikova O. (2019) Indeks elektricheskoj nestabil'nosti miokarda: klinicheskoe i prognosticheskoe znachenie. Rossijskij kardiologicheskij zhurnal, vol. 24 (12), pp. 55-61. doi: 10.15289/1560-4071-2019-12-55-61.

14. Harmon K., Suchslard I. (2020) Comparison of cardiovascular screening in athletes by history and physical examination with and without electrocardiogram: efficiency and cost. Heart Rhythm, vol. 17 (10), pp. 1649-1655. doi: 10.1016/j.hrthm.2020.04.032.

Подана/Submitted: 25.06.2021

Принята/Accepted: 18.10.2021

Контакты/Contacts: frolov.minsk@gmail.com 